\title{
EL CONOCIMIENTO Y SU RELACIÓN CON EL APRENDIZAJE DE LOS ESTUDIANTES EN LAS SOCIEDADES ACTUALES
}

Por

Fanny Mercedes González Pinzón ${ }^{1}$

E-mail.gopifame@hotmail.com

La nueva conceptualización entre educación y sociedad consiste en sustentar que el conocimiento es el factor clave del desarrollo de toda sociedad y el sostén del crecimiento económico y de sus relaciones sociales. Por esto se requiere de un aprendizaje que comprometa las actividades cotidianas del hombre, lo cual supone una nueva manera de pensar interpretar y de relacionarse con la realidad, natural y social. Se precisa la necesidad de revisar las diferentes teorías de aprendizaje, y su relación con el conocimiento que es enseñado en las instituciones educativas, esto nos permite mirar las maneras como los teóricos en educación explican el proceso que permite aprender para así caracterizar los marcos conceptuales que orientan las acciones educativas de maestros y estudiantes en las sociedades del presente. La reflexión se centra en las teorías constructivistas de la educación.

\section{El conocimiento}

Los conocimientos, ideas, concepciones, valores y creencias que caracterizan la vida de los estudiantes y docentes son el resultado de sus vivencias y de su participación en los diferentes procesos sociales y culturales en los cuales han estado inmersos. Así se asumen posturas ideológicas en la sociedad, que están en relación con la capacidad del ser humano de interpretar su realidad natural y social y en particular con la forma como explican y se explican la naturaleza del conocimiento $y$, en consecuencia, en la medida de lo posible, tratan de actuar siendo consecuentes con estas.

Las concepciones mecanicistas, desde tiempos de la antigüedad, conciben el conocimiento como una copia de la realidad - el conocimiento se considera el reflejo de ese mundo externo que existe con 
independencia del observador- Sobre la forma como se aprende el conocimiento se precisa que este, es un bien que debe ser entregado al estudiante en la práctica educativa preestablecida.

La creencia que al emitir una información por parte del profesor se genera una asimilación por parte del estudiante, hoy en día está siendo replanteada. Desde esta postura, el acto de aprender simplemente es, el de descubrir la realidad, la cual es considerada como única, externa y objetiva al sujeto, y se basa en la observación de sucesos y cosas. La concepción que subyace en esta actividad evidencia una relación mecánica entre transmisión y asimilación, situación que ha llevado a la realización de actividades que privilegien la memorización y repetición.

A partir de la epistemología en su versión actual, «el conocimiento deja de ser concebido como representación de la realidad externa $y$, en su lugar, es visto como resultado inseparable de las experiencias del sujeto y de su actividad cognoscitiva. El sujeto deja de ser cognitivamente pasivo frente al objeto del conocimiento. ${ }^{2}$

En este proceso la realidad es subjetiva, se encuentra sujeta a la conciencia y experiencia del sujeto, en consecuencia se pueden dar consensos $e$ interpretaciones distintas entre los sujetos, así el acto de conocer se concibe como un proceso adaptativo que organiza el mundo, no conduce al descubrimiento de un mundo independiente o preexistente fuera de la mente del que aprende. Por eso se afirma que el conocimiento se construye activamente por sujetos cognoscentes, no se recibe pasivamente del ambiente. ¿Con qué instrumentos realiza la persona dicha construcción? Fundamentalmente con los esquemas que ya posee, es decir, con lo que ya construyó en su relación con el medio que le rodea. ${ }^{3}$

Desde esta reflexión es que Piaget elabora su teoría sobre el problema del conocimiento a partir de la perspectiva constructivista, desde la cual señala que el problema del conocimiento ya no es qué conocemos o cómo conocemos sino cómo construimos nuestras interpretaciones del mundo, y qué mundo surge de esas interpretaciones. ${ }^{4} \mathrm{Su}$ teoría epistémica centro su atención en dar repuesta a la pregunta: ¿Cómo se pasa de un estado de menor conocimiento a un estado de mayor conocimiento?

\section{Las teorías de aprendizaje}

En la práctica educativa todo docente adopta manera implícita o explícita una o varias teorías de aprendizaje o elabora una integración de diversas teorías ayudado por sus experiencias, su formación, sus valores y sus creencias, que constituyen la concepción desde la cual el educador modela y construye sus estrategias para lograr el aprendizaje en sus estudiantes. Sin embargo muchas veces no es consiente, si esa concepción que respalda sus estrategias de aprendizaje, es o no coherente con los propósitos de desarrollar aprendizajes $y$, que pueden ser la causa de los fracasos en el aprendizaje de sus estudiantes.

Margarita Castañeda define una teoría de aprendizaje como:

Un punto de vista sobre lo que significa aprender. Es una explicación racional, coherente, científica y filosóficamente fundamentada acerca de lo que debe 


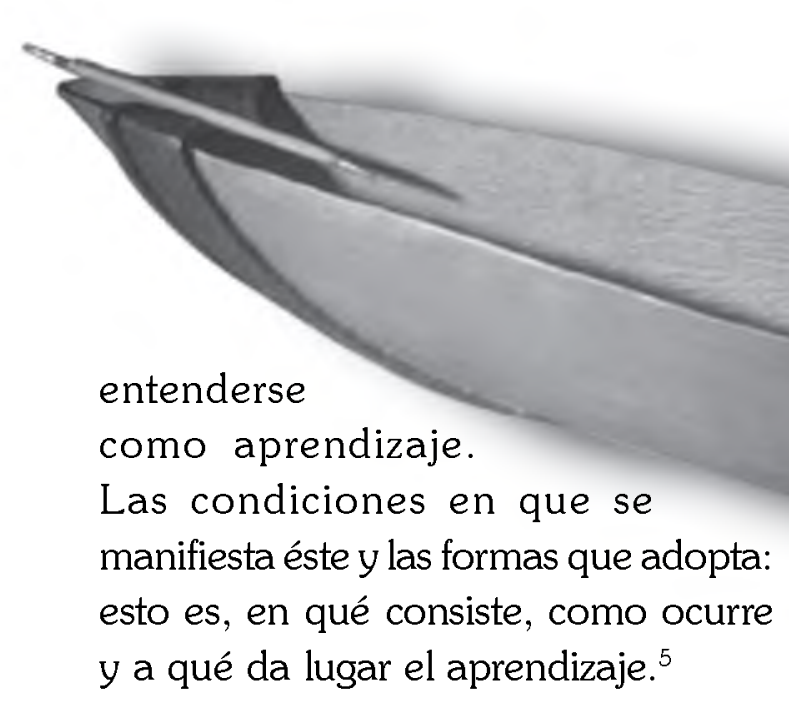

Es decir las teorías son un referente explicativo que permite definir una visión de trabajo en relación con las diferentes maneras como le damos respuesta a las acciones educativas que emprendemos cuando hacemos trabajo de aula.

Existen distintas corrientes teóricas sobre el aprendizaje entre estas tenemos: las corrientes conductivistas: representado por teóricos como Skinner, Wolpe, Salter, Gagné, y Smitth, etc. cuyo objetivo es el control y entrenamiento de la conducta. Según el conductismo el aprendizaje es el cambio de conducta duradero y observable que ocurre como resultado de sus experiencias.

Otra de las corrientes que ha despertado serías polémicas por la manera como se han querido traspolar sus pensamientos al aula, es el constructivismo, quizás por una inadecuada interpretación de este paradigma, desde el cual quieren legitimar practicas tradicionales. Las creencias de los docentes sobre el método tradicional del enseñar están tan afianzadas en los maestros que otras alternativas son percibidas como un no enseñar, razón por la cual, se nos sugiere ser mas reflexivos sobre nuestras

a c c i o nes

educativas.

En el ejercicio del enseñar tradicional, la tarea del maestro se limita a explicar los diferentes temas, y la del alumno a producir los conocimientos que recibe del profesor. Ahora se requiere de un docente que diversifique sus estrategias en función de los diferentes procesos de aprendizaje que promueve en el alumno, para que éste pueda alcanzar las metas propuestas.

Concebimos la concepción constructivista como el marco explicativo desde el cual es posible analizar cómo actuamos en la relación con nuestros estudiantes en el aula. Así el constructivismo es el conjunto articulado de principios y teorías que permiten explicar la naturaleza de las acciones de enseñanza y aprendizaje, o sea que estas teorías y principios nos van a facilitar instrumentos de análisis y reflexiones sobre la práctica educativa.

Frida Díaz Barriga nos habla de la existencia de dos grandes tradiciones constructivistas. Una sustentada en la disciplina pedagógica y otra de orden sociológico. La primera se centra en el estudio y funcionamiento del contenido de la mente (constructivismo sicogénetico de 
Piaget) la otra en el desarrollo de los dominios de orden social (constructivismo social de Vigotsky y la escuela socio cultural), mientras que para otros más, ambos aspectos son indisociables y perfectamente conciliables. También es posible identificar un constructivismo radical, planteado por autores como Von Glaserfeld o Maturana quienes postulan que la construcción del conocimiento es enteramente subjetiva. ${ }^{6}$ Entre esta serie de corrientes es que se ubica el debate actual de la psicología constructivista.

Para Cesar Coll la postura constructivista se alimenta de las aportaciones de diversas corrientes psicológicas asociadas no sólo al enfoque psicogenético piagetiano, sino también a algunos aportes de la psicología cognoscitiva que dan cuenta de la construcción de esquemas cognitivos, modelos mentales o teorías implícitas en los individuos; de la teoría ausbeliana de la asimilación y del aprendizaje significativo, del enfoque socio-cultural de Vigotsky y sus actuales seguidores incluido jerónimo Bruner, de las teorías de la atribución que explican la motivación del aprendizaje y otras teorías mas recientes. ${ }^{7}$

Así, en el debate constructivista sobre la educación se destacan tres enfoques: el enfoque psicogenético piagetiano, la psicología cognitiva y la corriente sociocultural vigotskyana.

Estos diferentes enfoques comparten el principio de la importancia de la actividad mental constructiva del alumno en la realización de los aprendizajes escolares. Este principio según Coll:
Es la idea fuerza más potente y también la más amplía compartida y trasladada al campo de la educación conduce a concebir el aprendizaje escolar como un proceso de construcción del conocimiento a partir de los conocimientos y de las experiencias previas y la enseñanza como ayuda a este proceso de construcción. Se logra aprender cuando se elabora una representación personal sobre el objeto, realidad o contenido que se pretende aprender. ${ }^{8}$

En este sentido el estudiante asume un compromiso con su propio proceso de aprendizaje. El es quien reconstruye los saberes de su grupo cultural, así será un sujeto activo el cual explora, investiga, descubre e inventa incluso cuando lee o escucha la exposición de otros.

Es así como para Piaget:

El sujeto se acerca al objeto del conocimiento dotado de ciertas estructuras cognitivas previamente construidas (no innatas) mediante las cuales asimila el objeto del conocimiento. Esta asimilación activa una transformación (acomodación) de su aparato cognitivo, de modo que en el siguiente acercamiento, su lectura del objeto será otra, pues como resultado de la primera, las estructuras cognitivas del sujeto se han modificado. ${ }^{9}$

Su argumento central es el esquema como unidad de análisis del pensamiento y es allí donde la acción de sujeto y el objeto de estudio son indisolubles gracias a los mecanismos de asimilación y acomodación. Si bien el proceso de construcción del 
conocimiento tiene un carácter individual, podríamos pensar en otras alternativas que den cuenta o complementen los proceso de construcción del conocimiento en el aula por el educando y hacemos referencia a los otros, los cuales también tienen un papel en este proceso, es así como sí aceptamos la necesidad de articular por una parte la dinámica interna de la actividad mental constructiva de los alumnos con la dinámica de las interacciones que se establecen en la escuela entre alumnos y docentes por medio del lenguaje o la actividad comunicativa, estaremos propiciando las condiciones para dar cuenta de los procesos de construcción del conocimiento en el aula.

De acuerdo a lo expuesto anteriormente el proceso de construcción del conocimiento va a depender de:

1. Los conocimientos y experiencias previas que poseen los estudiantes, los cuales son aprendidos en un proceso de educación informal o formal, cuando él interactúa en su trayectoria de vida.

2. De la representación que se tenga de la nueva información o de la actividad o tarea a resolver; representación que tiene que ver lo que el estudiante ha construido desde su cotidianidad y que se expresa en creencias, valores, opiniones. En ella parecen elementos informativos, cognitivos, ideológicos, normativos que permiten al estudiante $y$ al maestro interpretar su realidad desde sus referentes contextuales.

3. Y por último de la actividad externa (exoestructuración) o interna (autoestructuración) que se efectúe al respecto. Es decir el conocimiento depende de lo que los sujetos poseen a priori en el momento de la percepción. Por consiguiente se requiere de estudiantes y docentes una comprensión que va más allá de la reflexión, es decir una reflexión que implica una reflexión sobre la reflexión y que los teóricos llaman metacognición ${ }^{10}$. Esta categoría de la metacognición es una función superior que se desarrolla y que se utiliza como mecanismo o forma de aprendizaje. Implica la formación de procesos educativos que se caracterizan por un alto nivel de conciencia $y$ de control voluntario y que se utiliza para agilizar otros procesos cognitivos más simples. Por ejemplo, buscar estrategias para optimizar las funciones como memoria, capacidad de análisis de percepción, de comprensión, de decisión, de selección; entre otras.

Podemos Concluir que la construcción del conocimiento escolar es el proceso mediante el cual el estudiante es quien selecciona, organiza y transforma el conocimiento que recibe de diferentes fuentes, estableciendo relaciones entre esta información y los conocimientos previos que posee. Este proceso implica una reorganización interna de esquemas que se producen cuando entran en conflicto lo que el alumno ya sabe: los conocimientos y experiencias previas que tiene, con los nuevos conocimientos que aprende. Ello significa para un maestro promover la construcción de nuevos ambientes propicios para la formación individualizada del estudiante en la cual, el propio estudiante, puede tomar decisiones en cuanto a los ritmos, modos y tiempos en que cursará los programas educativos de acuerdo a sus necesidades, características, antecedentes académicos y posibilidades de dedicación, generando así un sentido de 
responsabilidad, libertad y autonomía. Así es necesaria una educación centrada en el estudiante la cual estará orientada por métodos y técnicas didácticas pedagógicas que pongan en el centro de atención al sujeto que aprende.

De acuerdo con la corriente socio-cultural Vigoskiana, la educación es una de las fuentes más importantes del desarrollo ontogenético en los miembros de la especie humana. El desarrollo que experimentan los seres humanos desde el nacimiento hasta la muerte es producto de las interacciones que se establecen entre el sujeto que aprende y los sujetos mediadores de la cultura, entre los cuales destacamos los educadores padres y compañeros, así como los códigos, mensajes simbólicos, propagandas, programas de medios de comunicación y maneras y modos de expresión cultural política y económica de las personas que forman parte de la sociedad.

Los postulados en los que se centra su explicación son:

La ley de la doble formación de los procesos psicológicos superiores y la educación como fuerza creadora $e$ impulsadora

d e 1 desarrollo: en el desarrollo cultural del niño, toda función aparece dos veces: primero, a nivel social, y más tarde, a nivel individual; primero, entre personas (interpsicológico) y después en el interior del propio niño» (intrapsicológica) ${ }^{11}$

Las funciones mentales superiores que son parte de la herencia social y cultural del niño van del plano social al psicológico, del interpsíquico al intrapsíquico, del plano de lo socialmente regulado a lo autorregulado. El desarrollo de la capacidad de aprendizaje del individuo, gradualmente y con el tiempo, necesitará cada vez menos ayuda para desempeñarse. El aprendizaje se logra cuando se da una influencia reciproca entre el sujeto y el medio social. Uno de los conceptos más importantes de Vigosky es el de la Zona de Desarrollo Próximo (ZDP) la cual la definió como:

La distancia entre el nivel de desarrollo efectivo o real, determinado por la capacidad de resolver un problema independientemente, y el nivel de desarrollo potencial, determinado a través de la resolución de un problema bajo la guía de un adulto o en colaboración con otro compañero más capaz. $^{12}$
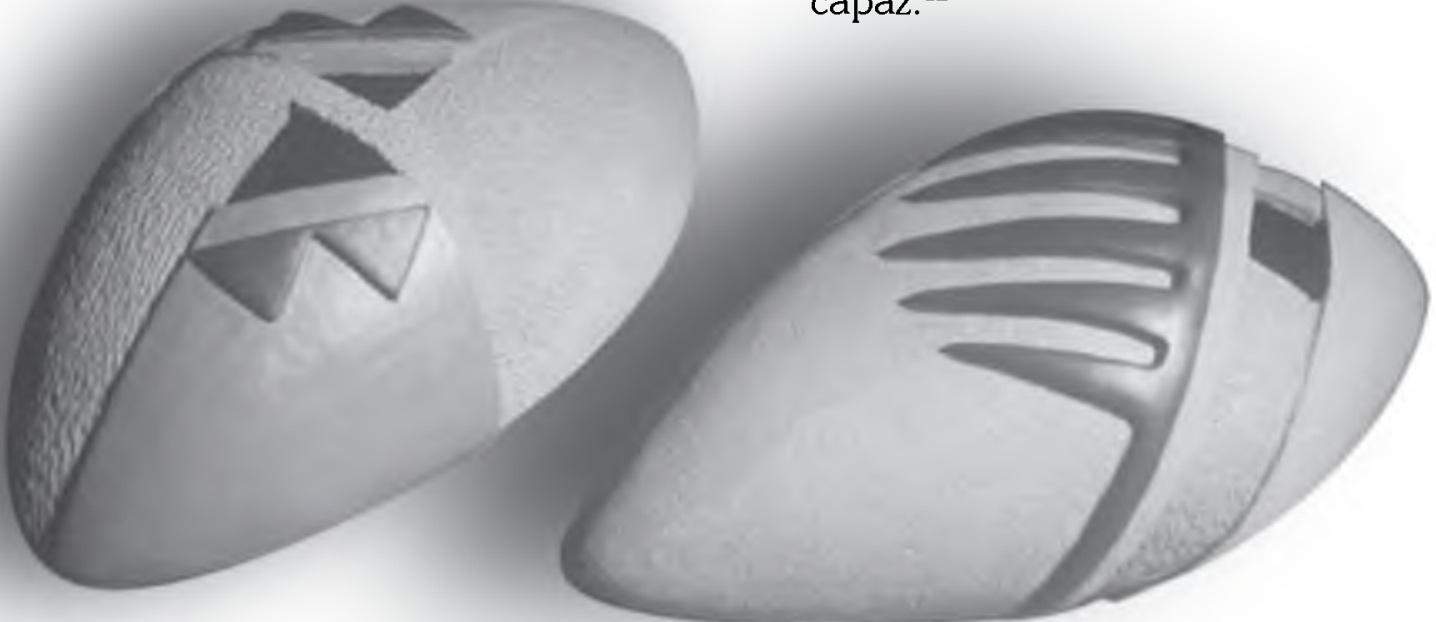
Se puede decir, entonces, que aprendemos cuando acompañamos al otro a través de la Zona de desarrollo Próximo, así por ejemplo el aprendizaje de un joven puede estar acompañado de la ayuda de otro más capaz, un compañero o el maestro, en un proceso continuo, ayudado por el otro y por sí mismo hasta lograr adquirir de manera independiente la nueva capacidad o aprendizaje. En este proceso son fundamentales las relaciones que se establecen entre Profesores y los alumnos, los acuerdos a los que se puedan llegar, las situaciones de enseñanza y contexto educativo. Es en la interacción donde se negocian y se construyen significados.

Es decir que este proceso de construcción de significados, y de construcción del conocimiento es un acto social no sólo personal, se realiza en interacción con los otros. Así la comunicación entre compañeros y docentes permite una comprensión mas profunda sobre las diferentes situaciones de aprendizaje que se desarrollan en el acto comunicativo, debido precisamente, a que cada sujeto tiene una manera distinta de ver la situación de enseñanza, que le permite construirla y enriquecerla en la medida en que posee una información anterior, unas experiencias, unas creencias y unos valores que hacen parte de sus vivencias.

El docente mas que dejar las tareas de enseñanza a los alumnos los acompaña propiciando las condiciones para que sean los mismos alumnos constructores reflexivos de su propio aprendizaje por tanto es necesario que el maestro presente las condiciones pedagógicas para desarrollar una educación pertinente, con sentido y significado culturalmente. Una educación pertinente socialmente es aquella en la que el proceso educativo se proyecta a la realidad del entorno y el momento histórico y cultural, cuando considera para su actuar académico las prioridades, exigencias y necesidades de las comunidades de la región. Tiene en cuenta la flexibilidad a las condiciones cambiantes del entorno, la criticidad constructiva y reflexiva frente a normas $y$ saberes de una disciplina, cuando se fomenta la capacidad de auto actualizarse permanentemente, cuando se recrea el conocimiento al servicio de la sociedad, cuando el estudiante es respetuoso y solidario con el desarrollo social y cultural. Todo lo anterior conduce la formación de individuos comprometidos con el bienestar de la sociedad y el entorno y con una actitud de respeto al otro y democrática.

Así mismo es preciso, que el maestro conozca los contextos sociales de los alumnos a quienes va acompañar, motivos y metas personales, la disposición por aprender, de tal manera que se aproxime a las características del entorno natural y humano, al mundo del trabajo, al contexto social, económico y cultural, a los desarrollos científico tecnológicos de hoy. Conocer los contextos sociales de los estudiantes, no sólo es entender las variables cuantitativas de los estudios tradicionales de diagnósticos sociales; es adentrase en la comprensión e interpretación de las realidades sociales dentro de las cuales el estudiante vive su cotidianidad. Es trabajar sobre el manejo de los distintos símbolos sociales, relacionados con el poder y las organizaciones que allí funcionan. Es conocer las características históricas y geográficas de las familias y comunidades con las cuales los alumnos han construido su saber social desde su 
cotidianidad, un saber cuya representación simbólica varia entre alumnos, dada la diferencia entre familias y representaciones de esa misma realidad.

En forma similar si el docente tiene un manejo riguroso de los estatutos científicos de la disciplina, su experiencia pedagógica, mediante las cuales ha afianzado sus conocimientos, lo llevan a asumir determinadas posiciones que indican la manera como aplica la investigación como proceso de comprensión social de la realidad; de esta manera el docente asume la comprensión de las formas como los científicos construyen sus teorías permitiéndole al estudiante, en algunos casos, recorrer estos mismos procesos.

En relación con estas dos formas de saberes, el docente las articulan por ello es necesario la comprensión del entorno social de los estudiantes y la manera como esa comprensión de su contexto le permite al docente una enseñanza y un aprendizaje más contextualizado, así los estudiantes trabajaran en tareas auténticas y significativas culturalmente, y resolverán problemas contextualizados, con pertinencia y sentido a las necesidades del medio y del desarrollo individual y social.

\section{BIBLIOGRAFÍA}

Coll, Cesar E Isabel Sole. «Los profesores y a concepción constructivista» En El constructivismo en el Aula. Editorial Grao. 1993 pp. 16

Coll, C y Solé, I. «La relación Profesor alumno en el proceso de enseñanza aprendizaje». En Coll, C, J. Palacios y A. Marchesi. Psicología de la Educación II Madrid Alianza Psicología 1995; 302
Díaz Frida. El aprendizaje de la historia en el bachillerato. Procesos de Pensamiento y Construcción de conocimiento en profesores y estudiantes del CCH/UNAM. México. Ciudad Universitaria. 1998.

Castañeda, Margarita. 1987. p.183

García Rolando. Piaget y el Problema del conocimiento; p42

Moreno Armella, Luís E y Waldegg, Guillermina. La epistemología Constructivista y la didáctica de las ciencias: ¿Coincidencia o Complementariedad? En Investigación Didáctica. Cinvestav. IPN. 1998; 422

\section{NOTAS}

(1) Economista Universidad Cooperativa de Colombia. 1983. Magíster en Educación Línea formación docente Universidad Autónoma del estado de Morelos México. Investigadora de la Red de Investigación Prospectiva en Educación Latinoamericana (IPIEL).

(2) Moreno Armella, Luís E y Waldegg, Guillermina. La epistemología Constructivista y la didáctica de las ciencias: ¿Coincidencia o Complementariedad? En Investigación Didáctica. Cinvestav. IPN. 1998; 422

(3) Carreto M. Limón M. 1993; 21. En Díaz, Barriga, Fryda. El aprendizaje de la historia en el Bachillerato: procesos de pensamiento $y$ construcción del conocimiento en profesores y estudiantes del $\mathrm{CCH} / \mathrm{UNAM}$. México. Ciudad Universitaria. 1998; 25. 
(4) García Rolando. Piaget y el Problema del conocimiento; p42

(5) Castañeda, Margarita. 1987. p.183

(6) Díaz Frida. El aprendizaje de la historia en el bachillerato. Procesos de Pensamiento y Construcción de conocimiento en profesores y estudiantes del CCH/UNAM. México. Ciudad Universitaria. 1998.

\section{(7) Coll César 1996}

(8) Coll, Cesar E Isabel Sole. «Los profesores y a concepción constructivista» En El constructivismo en el Aula. Editorial Grao. 1993 pp.16

(9) Moreno Armella, Luís E y Waldegg, Guillermina. La epistemología Constructivista y la didáctica de las ciencias: ¿Coincidencia o Complementariedad? En Investigación Didáctica. Cinvestav. IPN. 1998;422

(10) Se define la metacognición como el conocimiento que tienen las personas sobre sus propios procesos cognitivos y las actividades necesarias para controlarlos. En la Pedagogía. Universidad Camilo José Cela. Editoral Espesa Calpe. España.2002;198

(11) Coll, C y Solé, I. «La relación Profesor alumno en el proceso de enseñanza aprendizaje». En Coll, C, J. Palacios y A. Marchesi. Psicología de la Educación II Madrid Alianza Psicología 1995;302

(12) Coll, Cesar e Isabel Sole.»Los profesores y la concepción constructivista. En César Coll, et al, El constructivismo en el aula. Barcelona. Editorial grajó. 1993; p18) 


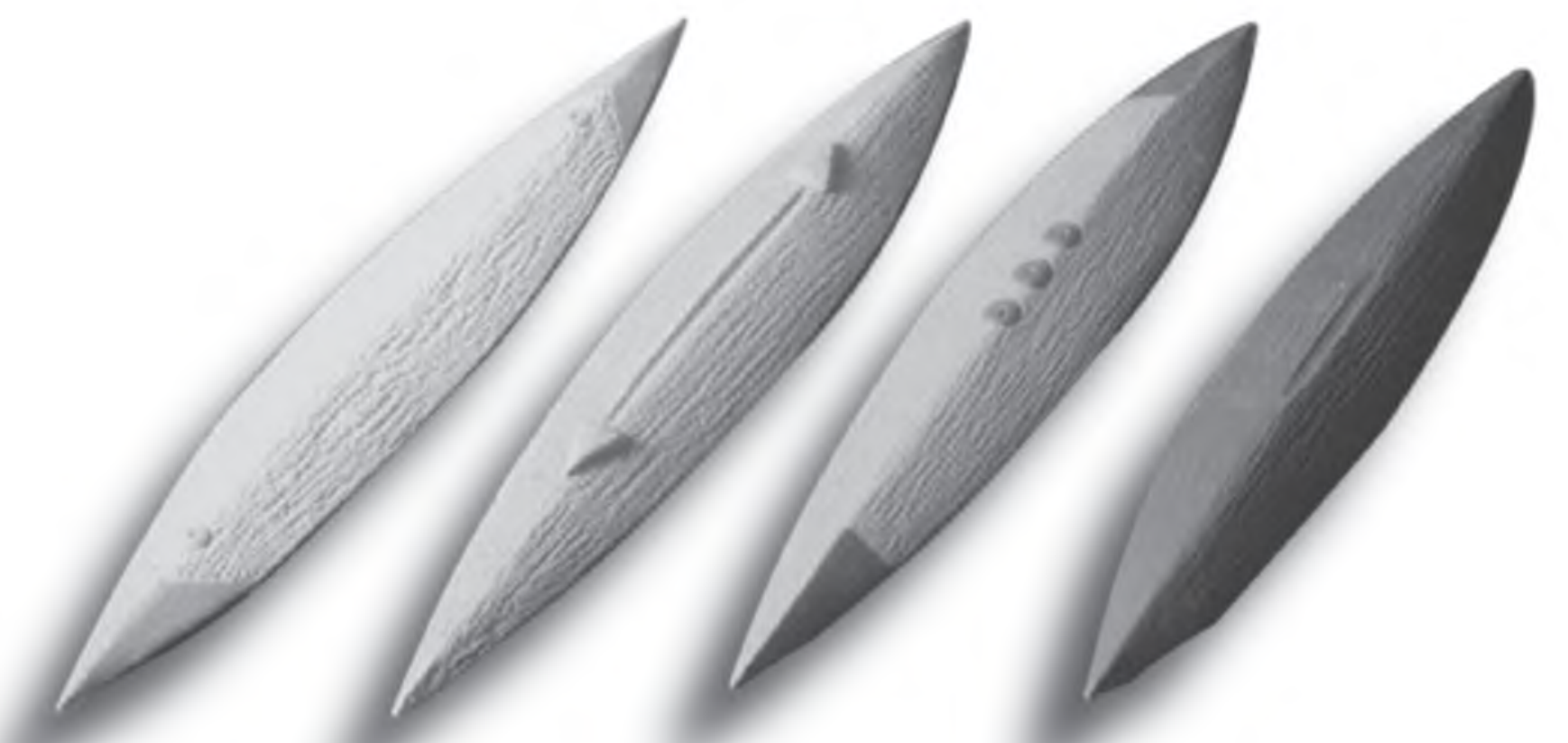

